\title{
A survey on antiemetics prescribed for children in some selected districts of Sri Lanka
}

\author{
S Sri Ranganathan ${ }^{1}$, K Mayurathan ${ }^{2}$, R Fernandopulle ${ }^{3}$ \\ Sri Lanka Journal of Child Health, 2010; 39: 93-97
}

(Key words: Domperidone, anti-emetic, drug utilization, children)

\begin{abstract}
Objective: To describe the prescription patterns of antiemetics in children in some selected districts of Sri Lanka and describe standard drug utilization variables, potential drug interactions, extent and types of irrational prescribing and rate of off-label use of anti-emetics.

Design, setting and methods: Analysis of paediatric prescriptions containing at least one antiemetic dispensed in eight community pharmacies (4 State, 4 Private) located in four different Provinces of Sri Lanka was performed over a period of four weeks by using a structured pre-tested checklist. Standard drug utilization variables including extent and types of irrational prescription were studied. .
\end{abstract}

Results: One hundred and eighty five prescriptions met the criteria; Domperidone was most frequently (89\%) prescribed followed by promethazine $(10.5 \%)$ and metoclopramide $(0.5 \%)$. Mean age was 4.48 years. Oral liquid dosage-form was prescribed in $54 \%$ followed by tablets $(32.5 \%)$ and suppositories $(11 \%)$; in $12 \%$ the dosage-form was unsuitable for age. Mean dose was $6 \mathrm{mg} ; 29.5 \%$ of the prescriptions carried irrational doses; $30 \%$ of prescriptions were off-label. Potential drug interactions with comedications were possible in $17.6 \%$ of instances. Majority of these deficiencies in prescription were observed in children younger than six years.

Conclusions: Domperidone was the most frequently dispensed anti-emetic for children in the selected districts. Irrational use of domperidone is more

\footnotetext{
${ }^{1}$ Senior Lecturer in Pharmacology and Consultant Paediatrician, Faculty of Medicine, University of Colombo, ${ }^{2}$ Medical Officer, Castle Street Hospital for Women, Colombo, ${ }^{3}$ Professor in Pharmacology, Faculty of Medicine, University of Colombo.
}

(Received on 24 July 2009. Accepted after revision on 18 September 2009) prevalent in young $(<6$ years) compared to older children.

\section{Introduction}

Vomiting is a common symptom of diverse aetiology and adversely affects the wellbeing of many children. Though in most instances vomiting could resolve spontaneously or with the treatment of aetiology, symptomatic treatment of vomiting in children is important. It reduces anxiety in the child and parents, allows successful administration of drugs, oral rehydration salts and other fluids. Hence, antiemetics are considered an effective adjuvant treatment in acute gastroenteritis

\section{Objectives}

- To determine the commonly utilized anti-emetics in children in some selected districts of Sri Lanka.

- To describe standard drug utilization variables, potential drug interactions, extent and types of irrational prescribing and rate of off-label use of anti-emetics.

\section{Methods}

Study design: Cross sectional descriptive study

Study setting: Pharmacies were selected according to convenient sampling. Four Osusala outlets of the State Pharmaceutical Corporation (SPC) which had pharmacists trained in drug utilization data collection (Colombo 7, Nugegoda, Kurunegala and Kandy) and 4 private pharmacies paired for each Osusala Pharmacy where the pharmacists were willing to get the training (Colombo 4, Colombo 14, Kurunegala and Kegalle) were selected for the study.

Study population and inclusion criteria: All prescriptions containing at least one anti-emetic (domperidone, metoclopramide, promethazine, 
ondansetron and granisetron) dispensed for children under the age of 12 years during the study period.

Study period and duration: Data was collected from all eight pharmacies for a period of four weeks from $26^{\text {th }}$ July to $25^{\text {th }}$ August 2007

Data collection instrument: A structured check list was designed to collect the required data on; (i) name of anti-emetic (ii) whether prescribed in generic or brand name (iii) brand name (iv) dosage form, dose, frequency and duration of anti-emetic treatment (v) quantity and cost of anti-emetic and (vi) names of comedications from prescriptions included for study.

Pre-testing data collection instrument: This checklist was pre-tested and necessary changes were done.

Data collection: Written prior permission was obtained from Authorities of SPC, branch managers of Osusala outlets and owners of private pharmacies included in the study. One pharmacist from each pharmacy was identified to collect the required data from prescriptions. All 8 pharmacists were trained by the investigators in using check list to collect data. Pharmacists at Osusala had prior experience in drug utilization data collection. Most data could be collected from the prescription itself; however in some instances if the required data was not available in the prescription (e.g. indication) study pharmacists were instructed to get the information from the person who brought the prescription. Contact details of the investigators were given to the pharmacists if there was a query. Investigators also regularly contacted the study pharmacists to monitor the data collection procedures.

Data analysis: At the end of the study period, the check lists were collected from the study pharmacists and the data was classified, coded, entered on to the Statistical Package for Social Sciences (SPSS) and analysed according to the objectives.

Assessment of suitability of dosage forms for paediatric age group: This was done according to WHO recommendations ${ }^{1}$. For example tablets were not considered suitable until 6 years of age.

Rationality of dose: Recommended dose $(\mathrm{mg} / \mathrm{kg})$ was taken from product information leaflet and cross checked with British National Formulary (BNF). Therapeutic dose for a child was determined by using this information and approximate weight of a Sri Lankan child for the age. Overdose, under dose and impractical (cannot be measured) dose were decided by comparing the therapeutic and prescribed dose.
Off label use: This is defined as when "a licensed medicine is used outside the conditions of the license" ${ }^{2}$. For the purpose of this study, off label use included use of a drug in situations not covered by the product license or summary of product characteristics i.e. at a different dose or frequency, in different clinical indications, in different age groups, administration by an alternative route, or in a formulation not approved for use in children.

Drug interaction: Potential drug interactions between the anti-emetic and the co-medications were determined by referring British National Formulary ${ }^{3}$ and searching the literature.

Irrational prescriptions: A medicine is irrationally used if it is prescribed when there is little likelihood that it will have a beneficial effect or when the anticipated benefit is not worth the potential harm or the cost of the drug ${ }^{4}$. It occurs when the medicine prescribed is incorrect, inappropriate, excessive, unnecessary or inadequate. We considered the following as irrational prescriptions; (i) prescriptions with inappropriate indications, (ii) prescriptions with over or under dose for the age, (iii) prescriptions given for longer than the recommended duration, (iv) prescriptions with unsuitable dosage forms for the age (v) prescriptions with co-medications which have potential drug interactions with the anti-emetic, and (vi) prescriptions which had co-medications contraindicated for that age. We have not included the cost and whether they were prescribed in generic or brand name as criteria for irrational prescription.

Data analysis tools: $\mathrm{BNF}$, WHO recommendation on suitability criteria for dosage forms in children, product information leaflets of the anti-emetics, and Summary of Product Characteristics of the antiemetics were used in analysing the data.

Ethical issues: Prior permission was obtained from the authorities of the study settings to collect the data. Identity details of neither patients nor prescribers were collected. Identity of the study setting was kept confidential. Research proposal was submitted to the Ethics Review Committee of Faculty of Medicine, Colombo, and the investigators were informed that ethical approval is not required as the study amounts to an audit and participants are not identified.

\section{Results}

Description of study population: One hundred and eighty five prescriptions containing at least one antiemetic dispensed to children under the age of 12 years during the study period were included, 99 
$(53.5 \%)$ from the state pharmacy outlets and 86 $(46.5 \%)$ from private pharmacies.

Type of anti-emetics: Domperidone was identified as the most frequently (165 prescriptions, 89\%) prescribed anti-emetic for children under the age of 12 years in the community settings with very few prescriptions for promethazine (19 prescriptions, $10.5 \%$ ) and metoclopramide (1 prescription, $0.5 \%$ ). Three prescriptions contained more than one antiemetic agent. Anti-emetics were written in brand names in $152(82 \%)$ prescriptions. Altogether nine different brands of domperidone had been prescribed and 4 of them accounted for $65 \%$ of all prescriptions.

Indication: Anti-emetics were prescribed for the treatment of vomiting in 174 prescriptions (94\%). In the remaining eleven prescriptions, promethazine $(n=8)$ was given for allergic reactions and domperidone for sleep disturbances $(n=1)$ and gastrooesophageal reflux $(n=2)$. Almost half of the prescriptions $(47.4 \%)$ for promethazine were given for allergic reactions.

Age and gender: Mean age was 4.48 years $(\mathrm{SD}=$ 3.03 ) with male to female ratio of $1.4: 1$. Almost half the prescriptions were given for children aged between 1 and 5 years with one fifth given for children under the age of one year.

Suitability of dosage form for the age: Anti-emetics had been prescribed in different dosage forms, oral liquid dosage form in $54 \%$ of prescriptions followed by tablets $(32.5 \%)$ and suppositories $(11 \%)$. In 22 $(12 \%)$ prescriptions, the dosage form of the antiemetic was not suitable for the age of the child.

Prescribed dose of anti-emetics: Information on dose was not available for 8 prescriptions. Commonly prescribed doses were $5 \mathrm{mg}(33.5 \%), 2.5$ $\mathrm{mg}(24 \%)$ and $10 \mathrm{mg}(23 \%)$ with a mean dose of 6 $\mathrm{mg}(\mathrm{SD}=5)$. However, when prescribed dose was compared with recommended dose for age of child, $29.5 \%$ of prescriptions were irrational (Table 1 ).

Frequency and duration of anti-emetic treatment: Anti-emetics were mostly (62.2\%) prescribed three times a day. In $17 \%$ of prescriptions they were given to be taken when required. Mean duration of treatment was 3.9 days $(\mathrm{SD}=3.8)$ with a range of one day to one month. Anti-emetics had been given for two weeks or more in eight $(4.3 \%)$ prescriptions.
Table 1

Comparing the prescribed dose of anti-emetic with recommended dose for the age of the child

\begin{tabular}{|l|c|c|}
\hline Prescribed dose & $\begin{array}{c}\text { Number of } \\
\text { prescriptions }\end{array}$ & $\begin{array}{c}\text { Percentage } \\
(\mathbf{\%})\end{array}$ \\
\hline Under dose & 31 & 17 \\
\hline Over dose & 12 & 6.5 \\
\hline Impractical dose* & 11 & 6 \\
\hline Correct dose & 123 & 66.5 \\
\hline No information & 8 & 4 \\
\hline Total & $\mathbf{1 8 5}$ & $\mathbf{1 0 0}$ \\
\hline
\end{tabular}

* Doses like $1.8 \mathrm{mg}$ which cannot be obtained from

$5 \mathrm{mg} / 5 \mathrm{ml}$ syrup or $10 \mathrm{mg}$ tablets

Co-medications: Anti-emetic was prescribed alone in $26(14 \%)$ instances; $66(36 \%)$ had one other comedication, 44 (24\%) had two and 49 (26\%) had three or more. Oral rehydration salts (ORS) was prescribed together with anti-emetics only in 22 instances, less than anti-bacterial agents, paracetamol, antihistamines and bronchodilators (Table 2).

Table 2

Frequencies of the most commonly prescribed co-medications

\begin{tabular}{|l|c|}
\hline Co-medications & Frequency (\%)* \\
\hline Oral rehydration salts & $22(11.9 \%)$ \\
\hline Anti-bacterial agents & $68(36.8 \%)$ \\
\hline Anti-amoebic agents & $11(5.9 \%)$ \\
\hline Paracetamol & $51(27.6 \%)$ \\
\hline Bronchodilators & $31(16.8 \%)$ \\
\hline Antihistamines & $23(12.4 \%)$ \\
\hline $\begin{array}{l}\text { Non-steroidal anti- } \\
\text { inflammatory drugs }\end{array}$ & $18(9.7 \%)$ \\
\hline Total prescriptions & $\mathbf{1 8 5}$ \\
\hline
\end{tabular}

* Percentage will not add up to 100 as there was more than one co-medication in a prescription

Drug interactions: Of the 159 prescriptions which contained another drug in addition to anti-emetic, potential drug interaction with the prescribed antiemetic was possible in $28(17.6 \%)$. Most frequently observed potential drug interaction was domperidone and drugs which have anti-cholinergic properties (negate the action of domperidone) and domperidone and erythromycin (inhibits the metabolism of domperidone and increases the cardiac toxicity).

Off label prescribing: Seven prescriptions did not have the required information to assess whether they were off label prescriptions. Of the remainder 52 $(30 \%)$ prescriptions were off label. Further analysis showed that $71 \%$ of the off label use were seen in 
children under the age of 6 years. Table 3 gives the different types of off label prescriptions.

Table 3

Different types of off label prescriptions for anti-emetics for children in community setting

\begin{tabular}{|l|c|}
\hline Type & Number (\%) \\
\hline Not off label & $126(68 \%)$ \\
\hline Off label by overdose & $31(17 \%)$ \\
\hline Off label by under dose & $12(6.5 \%)$ \\
\hline Off label by age & $06(3 \%)$ \\
\hline Off label by duration & $02(1 \%)$ \\
\hline Off label by indication & $01(0.5 \%)$ \\
\hline Inadequate information & $07(4 \%)$ \\
\hline Total & $\mathbf{1 8 5}$ \\
\hline
\end{tabular}

Cost of anti-emetics: We only analysed the cost of domperidone as it was the most frequently prescribed anti-emetic in our study sample. Cost depends on dose, dosage form, frequency, duration and whether the product was generic or brand. The cost for domperidone therapy in our study sample ranged from 1.80 SLR to 831. 87 SLR (mean 118, SD = 119). Twenty-five prescriptions were written in generic name where the cost ranged from 3.56 SLR to 145.00 (mean $37.45, \mathrm{SD}=41.74$ ) and 140 were for brand products with cost ranging from 1.80 SLR to 831.87 SLR (mean 132.93, $\mathrm{SD}=123.02$ ).

Irrational prescribing: Eighty four (45.4\%) prescriptions for anti-emetics in our study sample were irrational. There were 113 irrational uses in those 84 prescriptions as some prescriptions were irrational in more than one criterion. Of the 113 irrational uses $76 \%$ occurred in children under the age of six years (Table 4).

Table 4

Types of irrational prescriptions of anti-emetics for children in the community setting

\begin{tabular}{|l|c|c|c|}
\hline \multirow{2}{*}{ Reasons for irrational use } & \multicolumn{2}{|c|}{ Frequency } \\
\cline { 2 - 4 } & $\mathbf{0 - 5}$ years & > 5 years & Total \\
\hline Inappropriate indication for anti-emetic & 1 & 0 & 54 \\
\hline Over/under/impractical dose for the age & 38 & 16 & 08 \\
\hline Longer than the recommended duration & 5 & 3 & 28 \\
\hline Potential drug interactions & 20 & 8 & 22 \\
\hline Unsuitable dosage forms for the age & 22 & 0 & $\mathbf{1 1 3 ( 1 0 0 \% )}$ \\
\hline Total irrational use & $\mathbf{8 6}(\mathbf{7 6 \%})$ & $\mathbf{2 7}(\mathbf{2 4 \%})$ & \\
\hline
\end{tabular}

Children younger than six years of age: Majority of deficiencies were observed in children younger than six years; all $100 \%$ of unsuitable dosage-forms, $71 \%$ of off-label uses, $65 \%$ of inappropriate doses, and $76 \%$ of irrational uses were observed in prescriptions dispensed to this age-group.

\section{Discussion}

Domperidone was the most frequently prescribed anti-emetic in children contributing to $89 \%$ with only one prescription for metoclopramide. This shows that domperidone is a widely prescribed anti-emetic for Sri Lankan children. WHO model list of medicines includes metoclopramide and not domperidone due to paucity of evidence to show that domperidone is the preferred antiemetic in children. Pharmacokinetically however, domperidone would be preferred in children because it does not readily cross the blood brain barrier and is less likely to cause central side effects $^{3}$. A case control study showed that 9 of 13 children with extra pyramidal symptoms, and 3 of 523 controls, had used metoclopramide for the treatment of vomiting associated with fever and/or influenza (odds ratio 390; 95\% confidence interval
61-2807) ${ }^{5}$. In Sri Lanka domperidone is included in the essential medicines list as paediatricians in Sri Lanka through clinical experience prefer domperidone to metoclopramide in children.

Important criteria observed by the WHO for essential drugs include that drugs should be available in appropriate dosage form and be of assured quality including bioavailability. Internationally, concerns have been raised that that many paediatric medicines are not available in suitable dosage forms and strengths ${ }^{1}$. However, in our study only $12 \%$ of the prescriptions had unsuitable dosage form which was restricted to prescribing tablets in children younger than six years. But this may be justifiable in certain instances in that tablets are cheaper than liquid preparations and a limited number of tablets can be bought instead of buying a whole bottle of liquid preparation for a short course of illness.

Results of this study show that about $30 \%$ of prescriptions had inappropriate doses. Domperidone dose ranges from $1.25-10 \mathrm{mg}$ depending on age of the child. Syrup has $5 \mathrm{mg}$ in $5 \mathrm{ml}$ and tablet strength 
is $10 \mathrm{mg}$. Hence prescribers could easily give the correct dose. The relatively higher prevalence of under dose compared to overdose raises a question as to whether child really benefited from the anti-emetic. Some doses such as $7 \mathrm{mg}$ and $3.8 \mathrm{mg}$ were practically impossible to administer. Prescriber should be aware of the recommended dose and the strengths of commonly available domperidone. Sometimes calculating dose according to body weight leads to impracticalities requiring adjusting to the most approximate appropriate dose.

Antibacterial agents, antihistamines, bronchodilators and oral rehydration salts (ORS) were the frequent comedications. It is explainable as acute gastroenteritis and upper respiratory tract infections are the most frequent causes of vomiting in children.

About $18 \%$ of prescriptions had co-medications which could have interacted with domperidone causing increased adverse effects or therapeutic failure. Hence, domperidone should not be considered a no-risk alternative to cisapride, which has been withdrawn from the market, as domperidone is known to possess electrophysiological properties similar to those of cisapride and class III anti-arrhythmic ${ }^{6}$ drugs. Erythromycin is known to inhibit the hepatic metabolism of domperidone and co-administration of these two medicines is contra-indicated. Of the 185 prescriptions studied, $15(8 \%)$ had these two medicines.

Many studies have documented the extent of use of unlicensed and off label drug preparations in children ${ }^{7,8}$. Prevalence of off label use in our study is about $30 \%$ as opposed to $10 \%$ of prescription given for children under primary care in the $\mathrm{UK}^{9}$. In certain instances, off label use in children is unavoidable, but in our study majority of off label use could have been prevented if a little more attention had been paid to calculating the dose.

Irrational use of medicine occurs when the medicine prescribed is incorrect, inappropriate, excessive, unnecessary or inadequate. We have shown the incorrect, inappropriate, excessive and inadequate use of domperidone. Unnecessary use cannot be determined by analysing the prescriptions only.

\section{Conclusions}

Domperidone was the most frequently dispensed antiemetic for children in selected districts. We have highlighted incorrect, inappropriate, excessive and inadequate use of domperidone in these prescriptions. Irrational use of domperidone was more prevalent in young ( $<6$ years) compared to older children.

\section{Acknowledgements}

Sincere thanks to (1) Secretarial staff of Department of Pharmacology, Faculty of Medicine, Colombo; (2) SPC Authorities: MD/CEO, Deputy General Manager /Marketing, Manager/ promotion, (3) Managers of Kurunegala, Kandy, Nugegoda and Town Hall Osusala outlets; and (4) Osusala and private pharmacy pharmacists who collected the data.

\section{References}

1. World Health Organization. The Selection and use of essential medicines. Technical Report Service 950. Geneva: WHO; 2007.

2. Licensing Medicines for children. Joint report of the British Paediatric Association and the Association of the British Pharmaceutical Industry. London: BPA; 1996.

3. British Medical Journal Publishing Group, Royal Pharmaceutical Society of Great Britain, and Royal College of Paediatrics and Child health. British National Formulary (BNF) for children. London: BMJ, RPSGB, RCPCH; 2007.

4. World Health Organization. The rational use of drugs. Report of the Conference of Experts. Geneva: WHO; 1985.

5. Menniti-Ippolito F. Traversa G. Da Cas R. Rossi R. Renna S. Barabino P. Saggiomo G. Vetrano F. Sagliocca L. Extrapyramidal reactions in children treated with metoclopramide. Italian Journal of Pediatrics 2004; 30(1): 49-52.

6. Drolet B, Rousseau G, Daleau P, Cardinal R, Turgeon J. Domperidone should not be considered a no-risk alternative to Cisapride in the treatment of gastrointestinal motility disorders. Circulation 2000; 102:1883-5.

7. McKinzie JP, Wright SW, Wrenn KD. Paediatric drug therapy in the emergency department: Does it meet FDA - approved prescribing guidelines? Am J Emerg Med 1997; 15:118-21.

8. Conroy S, Choonara I, Impicciatore P, Mohn A, Arnell H, Rane A, et al. Survey of unlicensed and off label drug use in paediatric wards in European countries. BMJ 2000; 320:79-82.

9. McIntyre I, Conroy S, Avery A, Corns H, Choonara I. Unlicensed and off label prescribing of drugs in general practice. Arch Dis Child 2000; 83:498-501. 